\title{
Phase Shift in an Atom Interferometer due to Spacetime Curvature across its Wave Function
}

\author{
Peter Asenbaum, ${ }^{1}$ Chris Overstreet, ${ }^{1}$ Tim Kovachy, ${ }^{1}$ Daniel D. Brown, ${ }^{2}$ Jason M. Hogan, ${ }^{1}$ and Mark A. Kasevich \\ ${ }^{1}$ Department of Physics, Stanford University, Stanford, California 94305, USA \\ ${ }^{2}$ School of Physics and Astronomy, University of Birmingham, Birmingham B15 2TT, United Kingdom
}

(Received 13 October 2016; published 1 May 2017)

\begin{abstract}
Spacetime curvature induces tidal forces on the wave function of a single quantum system. Using a dual light-pulse atom interferometer, we measure a phase shift associated with such tidal forces. The macroscopic spatial superposition state in each interferometer (extending over $16 \mathrm{~cm}$ ) acts as a nonlocal probe of the spacetime manifold. Additionally, we utilize the dual atom interferometer as a gradiometer for precise gravitational measurements.
\end{abstract}

DOI: 10.1103/PhysRevLett.118.183602

A long-standing goal in matter-wave interferometry has been to resolve a phase shift associated with spacetime curvature across a particle's wave function [1-7]. The conceptual significance of such a tidal phase shift $\left(\phi_{\text {tidal }}\right)$ arises from the fact that acceleration and spacetime curvature have different physical origins in general relativity: Local acceleration arises from nongravitational forces, while curvature characterizes the spacetime manifold [2]. Unlike phase shifts arising from local acceleration, curvature-induced phase shifts have been described as representing the first true manifestation of gravitation in a quantum system [1-5].

In prior gravitational measurements exploiting de Broglie wave interference [8-15], the interferometer arm separation was small enough that the spacetime curvature across the wave function (i.e., gravity gradient across the interferometer arms) did not produce an identifiable tidal phase shift. For the purpose of understanding gravitational effects in these experiments, the trajectory of each interfering particle is well described by a single geodesic that is defined by that particle's initial position and velocity before the interferometer. The interferometer phase measures the local acceleration of this geodesic relative to the interferometer beam splitters and mirrors.

To clarify this distinction, we consider as an example the case of light-pulse Mach-Zehnder atom interferometry with momentum transfer $n \hbar k$ and pulse spacing $T$. We denote the position (velocity) of a given particle $i$ at the time when the first beam splitter is applied as $z_{i}\left(v_{i}\right)$, the local gravitational acceleration at position $z_{i}$ as $g_{i}$, and the atomic mass as $m$. For simplicity, we consider a uniform gravity gradient $T_{z z}$. We initially consider the regime in which the interferometer arm separation is small enough that tidal forces across the arms can be neglected. With this assumption, we compute the phase shift for two different cases: first, with the lasers that generate the interferometer beam splitters and mirrors following the particle's geodesic and, second, with the lasers fixed in the lab frame. In the first case, the phase shift is zero. In the second case, the phase shift is $\phi_{\mathrm{lab}}=n k g_{i} T^{2}+n k v_{i} T_{z z} T^{3}+(7 / 12) n k g_{i} T_{z z} T^{4}$ [16]. Since the phase shift is zero when the lasers move along the particle's geodesic, the entire phase shift $\phi_{\text {lab }}$ can be understood as arising from the relative motion of the lasers and this geodesic [17]. This phase shift includes the effect of the coupling of initial conditions to the gravity gradient [10] but does not include $\phi_{\text {tidal }}$.

If the interferometer arm separation is made large enough that there are resolvable tidal forces across the spatial extent of the interferometer, then the wave function of an interfering particle can no longer be approximated as traveling along a single populated geodesic. Instead, the two arms follow separate trajectories that accelerate with respect to one another. A tidal phase shift can be observed in this regime-it appears as [16] $\phi_{\text {tidal }}=$ $(\hbar / 2 m) n^{2} k^{2} T_{z z} T^{3}$ in this example regardless of whether the lasers are fixed in the lab frame or follow the geodesic defined by the particle's initial position and velocity. From these considerations, $\phi_{\text {tidal }}$ is a manifestation of gravitational curvature that cannot be interpreted as simply arising from the relative motion of a single atomic trajectory and the interferometer lasers.

In this Letter, we report the first observation of a tidal phase shift. In our experiment, this phase shift is induced by a $84 \mathrm{~kg}$ proof mass in a geometry chosen so that the interferometer wave packet separation is comparable to the length scale of the change in the gravitational field. We employ large momentum transfer (LMT) light-pulse atom interferometry [18-21] and long interrogation times [22] to achieve wave packet separations in excess of $10 \mathrm{~cm}$. We identify $\phi_{\text {tidal }}$ by observing the interferometer phase shift as a function of wave packet separation and proof mass position.

The core apparatus has been described previously [22-24]. Each experimental run begins with the preparation of an ultracold ${ }^{87} \mathrm{Rb}$ atom cloud. The atoms are launched vertically into a $10 \mathrm{~m}$ tall atomic fountain using an optical 
lattice. Subsequently, the atoms are collimated by an optical dipole lens ( $1 \mathrm{~mm}$ waist, red-detuned laser beam) in the transverse dimensions. This lens is applied $\sim 100 \mathrm{~ms}$ after the end of the launch. Ultimately, the launched atom cloud contains $\sim 10^{6}$ atoms with an effective temperature of $\sim 50 \mathrm{nK}$ in the transverse dimensions.

A dual interferometer configuration $[13,20,25]$ is used to suppress spurious phase shifts arising from vibrations in the laser delivery optics (see Fig. 1). The LMT atom optics consist of sequences of absolute ac Stark-shift compensated, two-photon Bragg transitions [22]. Figure 1(a) illustrates the spacetime diagram associated with a single-source dual interferometer sequence [20]. An initial LMT beam splitter sequence splits the atom cloud into two wave packets with momenta differing by $N_{1}$ photon momentum recoil kicks $\left(N_{1} \hbar k\right.$, where $k$ is the wave number of the laser used to drive the Bragg transitions) in the vertical direction. The wave packets are allowed to freely drift apart for a time $\tau$. Next, the initially accelerated arm is decelerated by an LMT sequence so that the momentum splitting between the wave packets is reduced to $2 \hbar k$. The
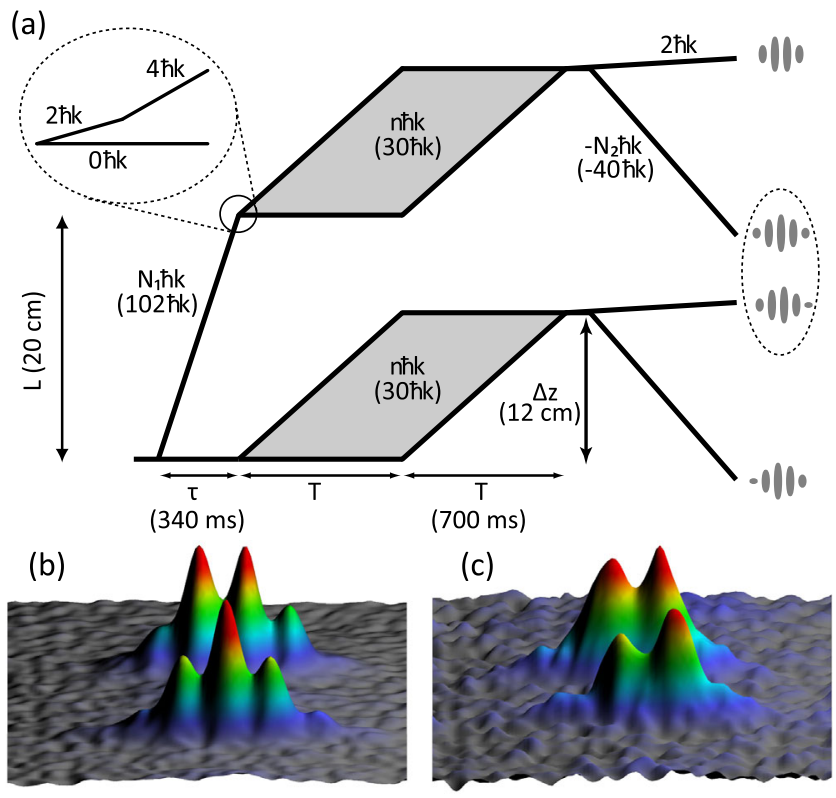

FIG. 1. (a) Spacetime diagram of the centers of the wave packets for a dual interferometer sequence, neglecting gravitational acceleration. An initial atom source is split into two clouds that drift apart with momentum difference $N_{1} \hbar k$ for time $\tau$, ultimately separating by the baseline $L$. Each cloud is used as the source for a Mach-Zehnder interferometer with momentum splitting $n \hbar k$ and pulse spacing $T$. The path separation between the two interferometer arms reaches a distance of $\Delta z=(n \hbar k / m) T$. The numerical values in parentheses indicate typical experimental parameters. In this work, $k$ is negative for the initial splitting and interferometer pulses; i.e., the interferometer trajectories are below the unperturbed launch height. Data plots: Fluorescence images of spatial interference fringes for an interferometer with (b) $\Delta z=4 \mathrm{~cm}$ $(n=10)$ and (c) $\Delta z=12 \mathrm{~cm}(n=30)$. two wave packets are vertically separated by a baseline $L=\left(N_{1} \hbar k / m\right) \tau$ and are the respective sources for the dual interferometers [26]. Before the initial beam splitter sequence, the vertical velocity distribution is filtered by two long-duration $\pi$ pulses (Gaussian temporal profile, FWHM $200 \mu \mathrm{s}$ ).

The interferometers are initiated by a beam splitter sequence like the one used to split the initial atom cloud (the two vertically displaced wave packets use opposite input ports of the first interferometer beam splitter, since their momenta differ by $2 \hbar k$ ). We use a Mach-Zehnder interferometer sequence with pulse spacing $T$. The momentum difference between the interferometer arms is denoted by $n \hbar k$. The laser system and optics configuration used to drive the Bragg transitions is described in Ref. [22].

We measure the differential phase shift between the two interferometers (gradiometer phase) by imaging one output port from each interferometer onto a CCD camera using resonant scattering. Because of the large vertical displacement between the two interferometers, we deliver an additional momentum kick $-N_{2} \hbar k$ to the lower port of each interferometer, so that the lower port of the upper interferometer and the upper port of the lower interferometer fit into the CCD camera's field of view at the time of detection [see Fig. 1(a)]. We use phase shear readout $[27,28]$ to extract a value for the gradiometer phase from each individual run of the experiment. Specifically, the angle of the Bragg laser beams is slightly tilted for the final beam splitter sequence using a piezo tip-tilt stage on the retroreflection mirror, imprinting a horizontal phase gradient across the cloud. This leads to horizontal spatial fringes in the interferometer output ports [see Figs. 1(b) and 1(c)], allowing for the single-shot determination of phase and contrast [27] in a single port. The relative phase between the two interferometers is then determined [29]. We implement measurements using interferometers with path separations $\Delta z$ of up to $\Delta z=16 \mathrm{~cm}$ with $L=20 \mathrm{~cm}$, $n=38$, and $T=700 \mathrm{~ms}$.

We placed several lead bricks near the apex of the interferometer trajectory and observed their effect on the gradiometer phase. The bricks produce a phase shift of $1.0 \mathrm{rad}$ [Figs. 2(a) and 2(b)], in agreement with the theoretical prediction obtained by numerically calculating the propagation, laser, and separation phases along the perturbed interferometer trajectories [16,30,31]. We find the systematic error of the gradiometer phase due to changes in the horizontal position of the atoms to be small [29]. Figure 2(c) compares the difference in the gradiometer phase (with and without bricks present) to its predicted value as a function of the launch height.

The macroscopic spatial and temporal scales of the interferometers allow the interferometers to resolve the tidal phase shift. The momentum recoil kicks that the atoms receive during the beam splitter and mirror interactions lead to wave packet trajectory deflections with a characteristic 

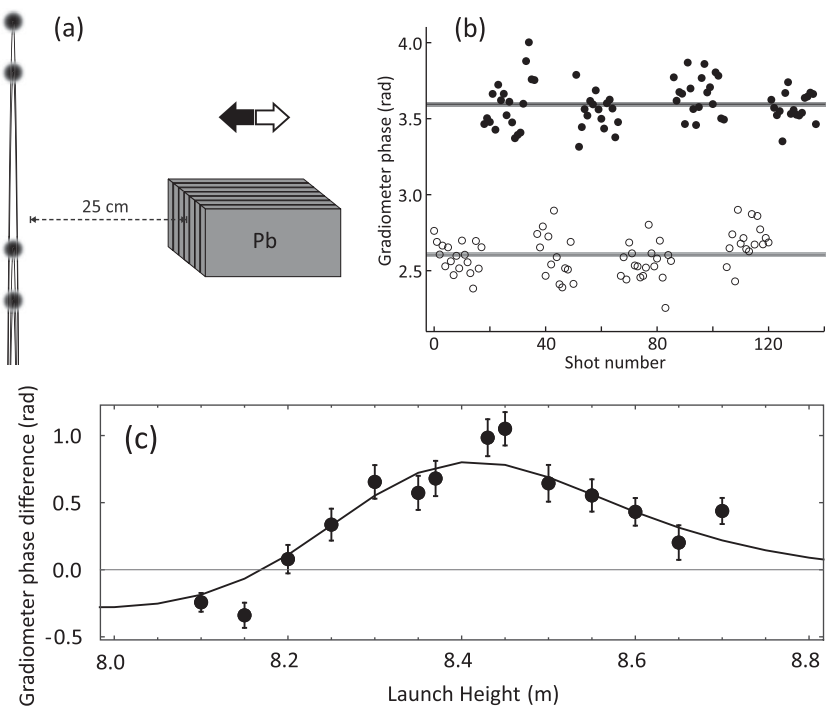

FIG. 2. (a) Schematic representation of the experimental setup for measuring the gravity gradient of seven lead bricks (total mass $84 \mathrm{~kg}$ ). (b) Measured gradiometer phase of a sequence with $h=8.45 \mathrm{~m}, L=32 \mathrm{~cm}, n=20$, and $T=600 \mathrm{~ms}(\Delta z=7 \mathrm{~cm})$, with (solid circles) and without (open circles) the bricks present. (c) Gradiometer phase difference (with and without bricks present) as a function of the launch height with $L=10 \mathrm{~cm}$, $n=30$, and $T=900 \mathrm{~ms}(\Delta z=16 \mathrm{~cm})$. The black, solid curve represents the full phase shift calculation.

size equal to the interferometer path separation $\Delta z=(n \hbar k / m) T$. Tidal forces over this distance scale lead to the tidal phase shift $\phi_{\text {tidal }} \simeq(\hbar / 2 m) n^{2} k^{2} T_{z z} T^{3}=$ $(1 / 2) n k \Delta z T_{z z} T^{2}$, which scales with the wave packet separation.

In a uniform gravity gradient, $\phi_{\text {tidal }}$ would be the same for the upper and lower interferometers and thus would not be present in the gradiometer phase. To circumvent this limitation, we use the gravitational field of the lead bricks, which has a spatially varying gradient [Figs. 3(a) and 3(b)]. The gradiometer phase difference therefore depends on the spatial variation of $T_{z z}$, and the difference in $\phi_{\text {tidal }}$ manifests as $\Delta \phi_{\text {tidal }} \simeq(\hbar / 2 m) n^{2} k^{2}\left(\Delta T_{z z}\right) T^{3}$ [14,32]. Figure 3(d) shows the difference between the gradiometer phase measured with and without bricks as a function of the LMTorder of the interferometer $n$. These measurements are compared to a theoretical model that does not include $\Delta \phi_{\text {tidal }}$. To remove $\Delta \phi_{\text {tidal }}$ from the calculation, we artificially consider trajectory deflections from the interferometer atom optics to be negligible (small-recoil trajectory). The small-recoil phase shift corresponds to the phase shift of the interferometer in the limit of highly massive interfering particles. The measurements deviate strongly from this model and agree with the model that includes the full phase shift calculation. Without the tidal contribution, the gradiometer phase difference would increase linearly with $n$ (see the discussion of $\phi_{\text {lab }}$ in the introduction) with the slope determined by the spatial variation of $g$ over the baseline $L$.
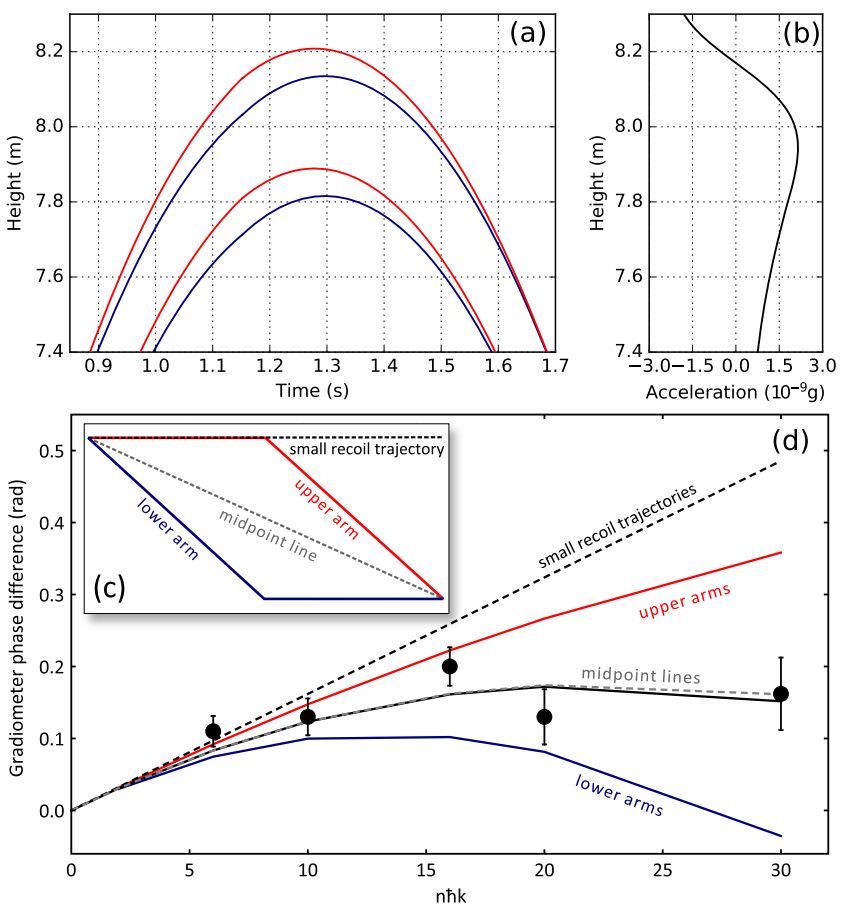

FIG. 3. (a) Spacetime diagram for a gradiometer with $h=8.25 \mathrm{~m}, L=32 \mathrm{~cm}, T=550 \mathrm{~ms}$, and $n=30$. (b) Calculated gravitational acceleration induced by the bricks as a function of the height along the interferometer axis. (c) Schematic of the interferometer trajectories, neglecting gravitational acceleration: upper (solid red lines) and lower (solid blue lines) arms, midpoint line (dashed gray line), and the trajectory without recoil from interferometer atom optics (dashed black line). (d) Gradiometer phase difference of a sequence with $h=8.25 \mathrm{~m}$, $L=32 \mathrm{~cm}$, and $T=550 \mathrm{~ms}$ as a function of $n(\Delta z=2 \mathrm{~cm}$ to $\Delta z=10 \mathrm{~cm})$. The black, dashed curve is the phase calculated without including the tidal phase shift. The solid red and blue curves are $\Delta \phi_{u}$ and $\Delta \phi_{l}$, respectively (see the main text). The gray, dashed (black, solid) curve is the phase predicted by the midpoint line (full phase shift) calculation. Each data point is the average of 15 shots.

The tidal phase shift is negligible unless the two arms of one interferometer experience accelerations that differ at the scale of the acceleration sensitivity - that is, there must be resolvable tidal forces across the wave function. To illustrate this, we separate the full phase shift into contributions representing the local gravitational acceleration along each interferometer arm. Labeling the two interferometers as $A$ and $B$, the phase shift of interferometer $\alpha \in\{A, B\}$ is approximately given by Bordé's midpoint theorem [33]:

$$
\begin{aligned}
\phi^{\alpha}= & n k\left(\frac{z_{u}^{\alpha}(0)+z_{l}^{\alpha}(0)}{2}\right)-2 n k\left(\frac{z_{u}^{\alpha}(T)+z_{l}^{\alpha}(T)}{2}\right) \\
& +n k\left(\frac{z_{u}^{\alpha}(2 T)+z_{l}^{\alpha}(2 T)}{2}\right) .
\end{aligned}
$$


Here $z_{u}^{\alpha}(t)$ is the position of the upper arm of interferometer $\alpha$ at time $t$, and $z_{l}^{\alpha}(t)$ is the position of the lower arm of interferometer $\alpha$ at time $t$. Note that $\phi^{\alpha}=1 / 2\left(\phi_{u}^{\alpha}+\phi_{l}^{\alpha}\right)$, where $\quad \phi_{u}^{\alpha} \equiv n k z_{u}^{\alpha}(0)-2 n k z_{u}^{\alpha}(T)+n k z_{u}^{\alpha}(2 T) \quad$ and $\phi_{l}^{\alpha} \equiv n k z_{l}^{\alpha}(0)-2 n k z_{l}^{\alpha}(T)+n k z_{l}^{\alpha}(2 T)$. Each of the quantities $\phi_{u}^{A}, \phi_{l}^{A}, \phi_{u}^{B}$, and $\phi_{l}^{B}$ corresponds to the phase shift of a Mach-Zehnder interferometer subject to the local acceleration along one interferometer arm.

We define the quantities $\Delta \phi_{u} \equiv \phi_{u}^{A}-\phi_{u}^{B} \quad$ and $\Delta \phi_{l} \equiv \phi_{l}^{A}-\phi_{l}^{B}$. At the experimental resolution, $\Delta \phi_{u}>\Delta \phi_{l}$ holds for a large enough wave packet separation [Fig. 3(d)], i.e., $\phi_{u}^{A}-\phi_{l}^{A}>\phi_{u}^{B}-\phi_{l}^{B}$. This indicates that, in at least one of the interferometers $A$ and $B$, the upper and lower arms experience resolvably different forces. Therefore, the phase shift of this interferometer is not determined by the local acceleration along a single populated trajectory, demonstrating that the atomic wave function is a nonlocal probe of the spacetime manifold [34].

The techniques demonstrated above are useful for applications involving precise measurement of gravitational gradients. The gradiometer achieves a resolution of $3 E$ per shot with parameters $L=32 \mathrm{~cm}, n=20$, and $T=600 \mathrm{~ms}\left(1 E=10^{-9} \mathrm{~s}^{-2}\right)$ [29]. This corresponds to a differential acceleration sensitivity of $1 \times 10^{-10} \mathrm{~g}$ per shot $\left(5 \times 10^{-10} \mathrm{~g} / \sqrt{\mathrm{Hz}}\right.$ given the $22 \mathrm{~s}$ cycle time $)$ and is near the estimated shot noise limit of $\sim 1 E$ per shot. Improvements in the atom source and imaging system would increase the atom number and contrast, allowing higher sensitivity, while a more advanced cold atom source [35] could reduce the cycle time to several seconds.

As an example of this capability, we measure the gravity gradient as a function of vertical position in the apparatus. This is done by varying the lattice launch velocity. Figure 4 shows the measured gravity gradient as a function of the launch height. The observed spatial variation of the gravity gradient is reasonably consistent with a model that includes the preliminary reference Earth model [36], the cylindrical pit surrounding the interferometer, and the basement in which the lab is located. An interpolation of the measured gravity gradient is used to predict the gradiometer phase as a function of launch height $h$ for other measurements, such as those described by Figs. 2 and 3.

Our approach differs from that employed by previous precision atomic gravity gradiometers [11-13], which used independently generated atom clouds separated by a baseline as the sources for two accelerometers. These gradiometers were subject to uncertainty in the baseline length due to source position fluctuations. In contrast, the baseline of the single-source gradiometer presented here is insensitive to the atom source position [37]. The idea of using LMT atom optics to create a single-source gradiometer was first demonstrated in a proof-of-concept experiment for a short baseline $(\sim 70 \mu \mathrm{m})[20]$ and has been proposed in the context of a space-borne gradiometer [39].
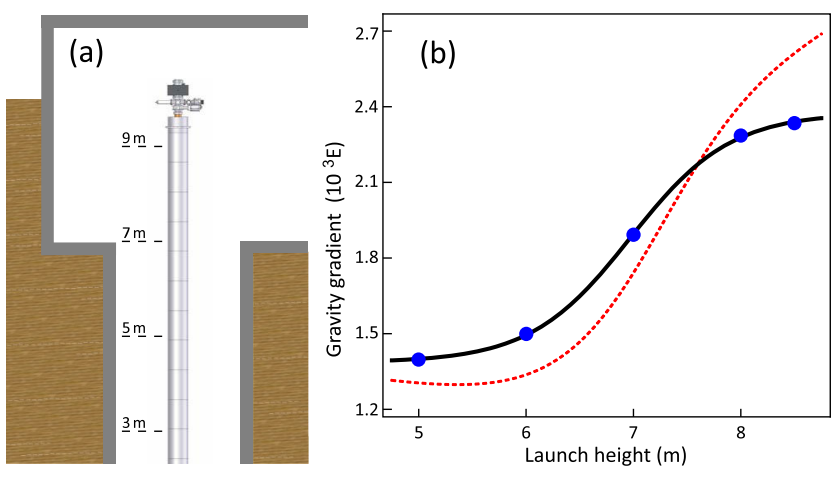

FIG. 4. (a) Schematic of the interferometer and surrounding mass, to scale, with indicated launch heights. (b) Measured gravity gradient (blue points) as a function of launch height $h$ for a gradiometer with $L=10 \mathrm{~cm}, n=10$, and $T=500 \mathrm{~ms}$. The red, dashed curve is a model of the interferometer response to the gravity gradient produced by a spherical Earth, including the effects of the cylindrical pit and basement in which the interferometer is located. The model has one free parameter, the density of Earth's surface near the interferometer, which fits to $\rho=2.3 \mathrm{~g} / \mathrm{cm}^{3}$. The black, solid curve is an interpolation (logistic fit, all parameters free). Each data point is the average of 50 shots. Statistical uncertainties are $\approx 6 E$ (error bars smaller than data points).

The observed phase stability of our gradiometer (e.g., $130 \mathrm{mrad}$ per shot for $L=32 \mathrm{~cm}, n=30$, and $T=550 \mathrm{~ms}$ ) can be used to constrain extensions of quantum mechanics that would manifest themselves through additional noise in the gradient measurement, due to, for example, anomalous wave packet localization at a length scale of $\sim 10 \mathrm{~cm}$ [40]. Bounding the phase noise of widely separated, macroscopic interferometers is complementary to previous work $[22,41,42]$, which was designed to be sensitive to spurious phase shifts that would occur inhomogeneously [22,42]. With a suitable source mass configuration, the dual interferometer could be used to measure the gravitational Aharonov-Bohm effect [43] and the gravitational constant $[44,45]$.

We thank M Squared Lasers for loaning us a SolsTiS laser. C. O. acknowledges support from the Stanford Graduate Fellowship. D. D. B. thanks the European Commission (EC) project Q-Sense (Project No. 691156) within the Horizon 2020 framework Quantum Hub for Sensors and Metrology (Engineering and Physical Sciences Research Council, EPSRC funding within Grant No. EP/ M013294/1). This work was supported by the Office of Naval Research, the Defense Threat Reduction Agency, and the Vannevar Bush Faculty Fellowship program.

[1] J. Anandan, Phys. Rev. D 30, 1615 (1984).

[2] J. Audretsch and K.-P. Marzlin, Phys. Rev. A 50, 2080 (1994).

[3] C. J. Bordé and C. Lämmerzahl, Phys. B1. 52, 238 (1996). 
[4] C. Chryssomalakos and D. Sudarsky, Gen. Relativ. Gravit. 35, 605 (2003).

[5] Y. Bonder and D. Sudarsky, Classical Quantum Gravity 25, 105017 (2008).

[6] K.-P. Marzlin and J. Audretsch, Phys. Rev. A 53, 312 (1996).

[7] B. Dubetsky, S. B. Libby, and P. Berman, Atoms 4, 14 (2016).

[8] R. Colella, A. W. Overhauser, and S. A. Werner, Phys. Rev. Lett. 34, 1472 (1975).

[9] M. Kasevich and S. Chu, Appl. Phys. B 54, 321 (1992).

[10] A. Peters, K. Y. Chung, and S. Chu, Nature (London) 400, 849 (1999).

[11] J. M. McGuirk, G. T. Foster, J. B. Fixler, M. J. Snadden, and M. A. Kasevich, Phys. Rev. A 65, 033608 (2002).

[12] F. Sorrentino, A. Bertoldi, Q. Bodart, L. Cacciapuoti, M. de Angelis, Y.-H. Lien, M. Prevedelli, G. Rosi, and G. M. Tino, Appl. Phys. Lett. 101, 114106 (2012).

[13] G. W. Biedermann, X. Wu, L. Deslauriers, S. Roy, C. Mahadeswaraswamy, and M. A. Kasevich, Phys. Rev. A 91, 033629 (2015).

[14] G. Rosi, L. Cacciapuoti, F. Sorrentino, M. Menchetti, M. Prevedelli, and G. M. Tino, Phys. Rev. Lett. 114, 013001 (2015).

[15] V. Nesvizhevsky et al., Nature (London) 415, 297 (2002).

[16] K. Bongs, R. Launay, and M. A. Kasevich, Appl. Phys. B 84, 599 (2006).

[17] M.-T. Jaekel, B. Lamine, and S. Reynaud, Classical Quantum Gravity 30, 065006 (2013).

[18] J. M. McGuirk, M. J. Snadden, and M. A. Kasevich, Phys. Rev. Lett. 85, 4498 (2000).

[19] H. Müller, S.-w. Chiow, Q. Long, S. Herrmann, and S. Chu, Phys. Rev. Lett. 100, 180405 (2008).

[20] S.-W. Chiow, T. Kovachy, H.-C. Chien, and M. A. Kasevich, Phys. Rev. Lett. 107, 130403 (2011).

[21] H. Ahlers, H. Müntinga, A. Wenzlawski, M. Krutzik, G. Tackmann, S. Abend, N. Gaaloul, E. Giese, A. Roura, R. Kuhl, C. Lämmerzahl, A. Peters, P. Windpassinger, K. Sengstock, W. P. Schleich, W. Ertmer, and E. M. Rasel, Phys. Rev. Lett. 116, 173601 (2016).

[22] T. Kovachy, P. Asenbaum, C. Overstreet, C. A. Donnelly, S. M. Dickerson, A. Sugarbaker, J. M. Hogan, and M. A. Kasevich, Nature (London) 528, 530 (2015).

[23] S. M. Dickerson, J. M. Hogan, A. Sugarbaker, D. M. S. Johnson, and M. A. Kasevich, Phys. Rev. Lett. 111, 083001 (2013).

[24] T. Kovachy, J. M. Hogan, A. Sugarbaker, S. M. Dickerson, C. A. Donnelly, C. Overstreet, and M. A. Kasevich, Phys. Rev. Lett. 114, 143004 (2015).
[25] M. J. Snadden, J. M. McGuirk, P. Bouyer, K. G. Haritos, and M. A. Kasevich, Phys. Rev. Lett. 81, 971 (1998).

[26] The finite duration of the optical pulses leads to small corrections to calculated distances.

[27] A. Sugarbaker, S. M. Dickerson, J. M. Hogan, D. M. S. Johnson, and M. A. Kasevich, Phys. Rev. Lett. 111, 113002 (2013).

[28] H. Müntinga et al., Phys. Rev. Lett. 110, 093602 (2013).

[29] See Supplemental Material at http://link.aps.org/ supplemental/10.1103/PhysRevLett.118.183602 for further details.

[30] P. Storey and C. Cohen-Tannoudji, J. Phys. II (France) 4, 1999 (1994).

[31] J. M. Hogan, D. M. S. Johnson, and M. A. Kasevich, in Proceedings of the International School of Physics "Enrico Fermi," edited by E. Arimondo, W. Ertmer, and W. P. Schleich (IOS Press, Amsterdam, 2009), pp. 411-447.

[32] In contrast to this work, the experiment described in Ref. [14] measured local accelerations at three heights to compute $\Delta T_{z z}$.

[33] C. Antoine and C. J. Bordé, J. Opt. B 5, S199 (2003).

[34] Bordé's midpoint theorem implies that the phase shift can also be approximately written in terms of the acceleration along the midpoint line, which is not a populated trajectory.

[35] J. Rudolph, W. Herr, C. Grzeschik, T. Sternke, A. Grote, M. Popp, D. Becker, H. Müntinga, H. Ahlers, A. Peters, C. Lämmerzahl, K. Sengstock, N. Gaaloul, W. Ertmer, and E. M. Rasel, New J. Phys. 17, 065001 (2015).

[36] A. M. Dziewonski and D. L. Anderson, Phys. Earth Planet. Inter. 25, 297 (1981).

[37] An alternative approach is to characterize the atom source position based on the position or time at which the atom cloud is detected [38].

[38] G. Tackmann, P. Berg, S. Abend, C. Schubert, W. Ertmer, and E. M. Rasel, C.R. Phys. 15, 884 (2014).

[39] O. Carraz, C. Siemes, L. Massotti, R. Haagmans, and P. Silvestrin, Microgravity Sci. Technol. 26, 139 (2014).

[40] S. Nimmrichter and K. Hornberger, Phys. Rev. Lett. 110, 160403 (2013).

[41] D. M. Stamper-Kurn, G. E. Marti, and H. Müller, Nature (London) 537, E1 (2016).

[42] T. Kovachy, P. Asenbaum, C. Overstreet, C. A. Donnelly, S. M. Dickerson, A. Sugarbaker, J. M. Hogan, and M. A. Kasevich, Nature (London) 537, E2 (2016).

[43] M. A. Hohensee, B. Estey, P. Hamilton, A. Zeilinger, and H. Müller, Phys. Rev. Lett. 108, 230404 (2012).

[44] J. B. Fixler, G. T. Foster, J. M. Mcguirk, and M. A. Kasevich, Science 315, 74 (2007).

[45] G. Rosi, F. Sorrentino, L. Cacciapuoti, M. Prevedelli, and G. M. Tino, Nature (London) 510, 518 (2014). 